\title{
Development and characterization of stabilized, polymerized phospholipid bilayers on silica particles for specific immobilization of His-tagged proteins
}

\author{
SEID M ADEM \\ Department of Chemistry, Washburn University, Topeka, Kansas, 66621, USA \\ e-mail: seid.adem@washburn.edu
}

MS received 29 May 2014; revised 23 October 2014; accepted 24 October 2014

\begin{abstract}
Stabilized phospholipid bilayer (PLB) coated silica microspheres were prepared via polymerization of lipid monomers. These lipid coated silica microspheres are stable to both extended storage in solution at $4^{\circ} \mathrm{C}$ and dry storage at room temperature. These stabilized lipid coated microspheres have also been functionalized with nickel-chelating lipids, a commonly used tool for immobilizing polyhistidine-tagged proteins. It is shown that 6xHis-EGFP interacts with (poly)bis-SorbPC/DOGS-NTA-Ni ${ }^{2+}$ coated silica and this interaction was interrupted by washing with imidazole indicating the reversibility of the interaction. No interaction was observed between the functionalized silica substrate and EGFP, which lacks the 6xHis-tag. Furthermore, these biocompatible (poly)bis-SorbPC coated microspheres were able to minimize non-specific protein adsorption.
\end{abstract}

Keywords. Stabilized phospholipid bilayer; functionalized silica; (poly)bis-SorbPC; flow cytometry; Histagged proteins.

\section{Introduction}

Liposomes derived from synthetic lipids have been deposited on solid surfaces and used as a model system for studying cell membrane events in vitro and as intracellular delivery vectors. ${ }^{1}$ Gilbert et al. ${ }^{1}$ developed a system consisting of lipid membranes deposited on surfaces of glass microspheres with diameters approximately $1.6 \mu \mathrm{m}$, similar in size as biological cells. These micrometer-sized lipid particle assemblies enable the architecture and properties of cell membranes to be mimicked, allowing the evaluation of the permeability of artificial membranes and their capacity to incorporate molecules of biological interest. Liposomes can readily be prepared in different $\mathrm{nm}$ size ranges using natural and synthetic phospholipids allowing the membrane properties to be tuned with respect to chemical functionalities, hydrophobicity of the lamellar region, lipid content, etc. Additionally, a number of important transmembrane receptors and transporters have been functionally reconstituted into liposomes, including GPCRs ${ }^{2-4}$ and ion channels. ${ }^{5-7}$

Lipid coated silica beads were also used as chromatographic supports for the separation of proteins. These new chromatographic supports developed by Bayerl and co-workers ${ }^{8}$ avoided protein denaturation and thus improved the classical techniques of size exclusion or ion exchange chromatographic techniques. Chromatographic columns were filled with porous silica beads that were covered with lipids. The lipid membrane in the separation column was a significant improvement over the use of bare particles since it offered a biocompatible surface that was capable of preserving the activity and conformation of the proteins. This new column also allowed collection of delicate proteins, which otherwise would be damaged during the separation process with conventional columns.

While liposomes are quite useful for many applications, particularly binding assays, they present a number of limitations. Fluid PLBs lack the desired chemical and mechanical stability to serve as long term biomimetic coatings on stationary phase materials. Moreover, fluid PLBs are readily damaged by brief exposures to common chemical and physical insults including air bubbles, exposure to organic solvents and surfactants.

In this report, I demonstrate the preparation and characterization of silica microspheres that have been coated with highly stabilized and functionalized phospholipid bilayers. Following assembly and stabilization of the phospholipid bilayers, these novel silica matrices were characterized in terms of stability by fluorescence microscopy and flow cytometry. The resulting silica microspheres are found to be stable to extended storage 
in solution at $4{ }^{\circ} \mathrm{C}$ and when kept dry at room temperature for two weeks. Furthermore, theses silica matrices have been functionalized using DOGS-NTA-Ni ${ }^{2+}$ lipids. This functionalization is an important technique in immobilizing proteins that have 6xHis tag in their structure. Fluorescence microscopy and flow cytometry are used to follow the interaction between $6 x$ HisEGFP and the functionalized silica microspheres. The interaction of the protein with the NTA-Ni ${ }^{2+}$ functionalized PLB-coated silica microspheres was specific and reversible upon the addition of a competitive ligand, imidazole.

\section{Experimental}

\subsection{Materials}

1,2-dioleoyl-sn-glycero-3-phosphocholine (DOPC), 1, 2-dipalmitoyl-sn-glycero-3-phosphoethanolamine-N(Lissamine Rhodamine B Sulfonyl)ammonium salt (Rh-DPPE) and (1,2-dioleoyl-sn-glycero-3- $\{$ [N(5-amino1-carboxypentyl)iminodiacetic acid]succinyl $\}$ nickel) (DOGS-NTA-Ni ${ }^{2+}$ ) were purchased from Avanti polar lipids (Alabaster, AL). Bis-SorbPC (1,2-bis[10-(2',4'hexadieoyloxy)decanoyl]-sn-glycero-2-phosphocholine was synthesized according to previous protocols. ${ }^{9}$ FM1-43 was obtained from Invitrogen Molecular Probes (Eugene, OR). Six-histidine tagged enhanced green fluorescent (6xHis-EGFP) protein was purified using Ni-NTA metal affinity chromatography from transected E. coli. Enhanced green fluorescent (EGFP) protein was obtained by cleaving the His-tag from 6xHis-EGFP using thrombin. HPLC grade acetonitrile (ACN) was from EMD Chemicals Inc. (Gibbstown, NJ). Silica microspheres were from Polysciences, Inc. (Warrington, PA). All aqueous solutions were prepared in water obtained from a Barnstead EasyPure UV/UF water purification system (Dubuque, IA) with a resistivity of $18 \Omega \mathrm{cm}$.

\subsection{Preparation of lipid coated silica microspheres}

Stock solutions of phospholipids used in this study were either in chloroform or benzene. The solvents were evaporated under Ar and lipids were further dried under vacuum overnight. The dried lipids were re-suspended in buffer and sonicated to clarity in a bath sonicator to obtain small unilamellar vesicles (SUVs). Lipid coated microspheres were prepared by adding $1 \mu \mathrm{m}$ or $5 \mu \mathrm{m}$ silica microspheres to the clear lipid solution and sonicated for $5 \mathrm{~min}$ and incubated for $30 \mathrm{~min}$ to allow vesicle fusion. The ratio of area of lipids to that of silica microspheres was approximately $25\left(\mathrm{~A}_{\text {lipid }} / \mathrm{A}_{\text {silica }} \approx 25\right)$. Then, the mixture was degassed for $10 \mathrm{~min}$ and polymerized with a UV pen lamp (Fisher Scientific, Denver, CO) while stirring for $30 \mathrm{~min}$. The lipid coated silica microspheres have been tested for stability studies by fluorescence microscopy and flow cytometry.

To study the interaction of microspheres with 6xHisEGFP, (poly)bis-SorbPC and DOPC coated microspheres were functionalized with DOGS-NTA-Ni ${ }^{2+}$, in which the PC and DOGS-NTA-Ni ${ }^{2+}$ lipid were mixed in a 95:5\% ratio by mole prior to lipid drying and buffer rehydration. For flow cytometry experiments, the PC lipids were mixed with Rh-DPPE in a 98:2\% ratio by mole, again prior to drying and resuspension with buffer.

\subsection{Stability studies}

Both polymerized and unpolymerized lipid coated silica microspheres were centrifuged and washed with $20 \mathrm{mM}$ PBS buffer at $\mathrm{pH} 7.4$, repeating four times to remove unbound and loose vesicles. The silica microspheres were then re-suspended with the buffer and a portion of the suspension was taken and incubated with FM143 for $30 \mathrm{~min}$., while the remaining was kept at $4^{\circ} \mathrm{C}$ for subsequent experiments. The reaction mixture with FM1-43 was diluted as needed for fluorescence imaging and flow cytometry experiments. On each subsequent day experiment was conducted, the same washing and centrifugation steps and incubation with FM1-43 were repeated. Similarly, for studies with functionalized lipid coated silica microspheres, the microspheres were washed and centrifuged with buffer for four cycles before imaging and flow cytometry experiments.

To study stability to dry storage, the lipid coated microspheres were washed and centrifuged following polymerization to remove unbound vesicles. After discarding the buffer following the final centrifugation, the microspheres were left open at room temperature to dryness till next experiment. When images were taken, the dried microspheres were re-suspended with buffer, washed thoroughly and centrifuged multiple times. Then, non-functionalized lipid coated microsphere suspensions were incubated with FM1-43 for 30 min and diluted as needed before imaging and flow cytometry experiments. These drying/rehydration steps were repeated for microspheres that have been kept open at room temperature for about two weeks.

\subsection{Fluorescence imaging}

Fluorescence images for lipids supported on silica microspheres were obtained with a Nikon Eclipse 
TE300 inverted epifluorescence microscope with 20x/0.5 and 4x/0.13 N.A objectives, respectively. MetaVue software (Universal imaging, Downingtown, PA) was used to capture and analyze the images.

\subsection{Flow cytometry}

Silica microsphere suspensions were analyzed by flow cytometry using a Becton-Dickinson FACScan flow cytometer (San Jose, CA) interfaced to a Power PC Macintosh. The FACScan is equipped with one argonion laser, three fluorescence channels and two channels for forward scatter and side scatter. The sample was illuminated at $488 \mathrm{~nm}$ and fluorescence signals were acquired through a 530/30 nm band pass filter. Data analysis was done using WinMDI version 2.8 software available from the internet.

\subsection{Non-specific protein adsorption}

Investigation of protein nonspecific adsorption was performed using 6xHis-EGFP incubated with bare and lipid coated silica microspheres for $30 \mathrm{~min}$. The suspensions of proteins with uncoated silica and lipid coated silica were then centrifuged. The particles were washed with buffer multiple times and centrifuged at least three times before the final suspensions were used for collecting fluorescence images and flow cytometry data.

\section{Results and Discussion}

\subsection{Stability studies}

3.1a Stability to extended storage at $4^{\circ} \mathrm{C}$ and exposure to organic solvent: The stability of (poly)bisSorbPC coated silica microspheres was studied for five months using fluorescence microscopy. Before imaging, the lipid coated silica was washed multiple times with a buffer with vortexing and centrifugation to get rid of unbound lipids. Then the lipid coated silica particles were treated with FM1-43. FM1-43 is a fluorogenic dye which becomes fluorescent upon staining lipid molecules. Figure 1 shows representative FM143 stained (poly)bis-SorbPC coated silica microsphere images immediately after fabrication and after storage for different times indicated in the figures. As can be seen from the figures, very bright and uniformly fluorescent microspheres were observed, even after being stored for extended period of time (figures 1, c-f). The control experiments with bare silica microspheres incubated with FM1-43 (figure S1A) or microspheres
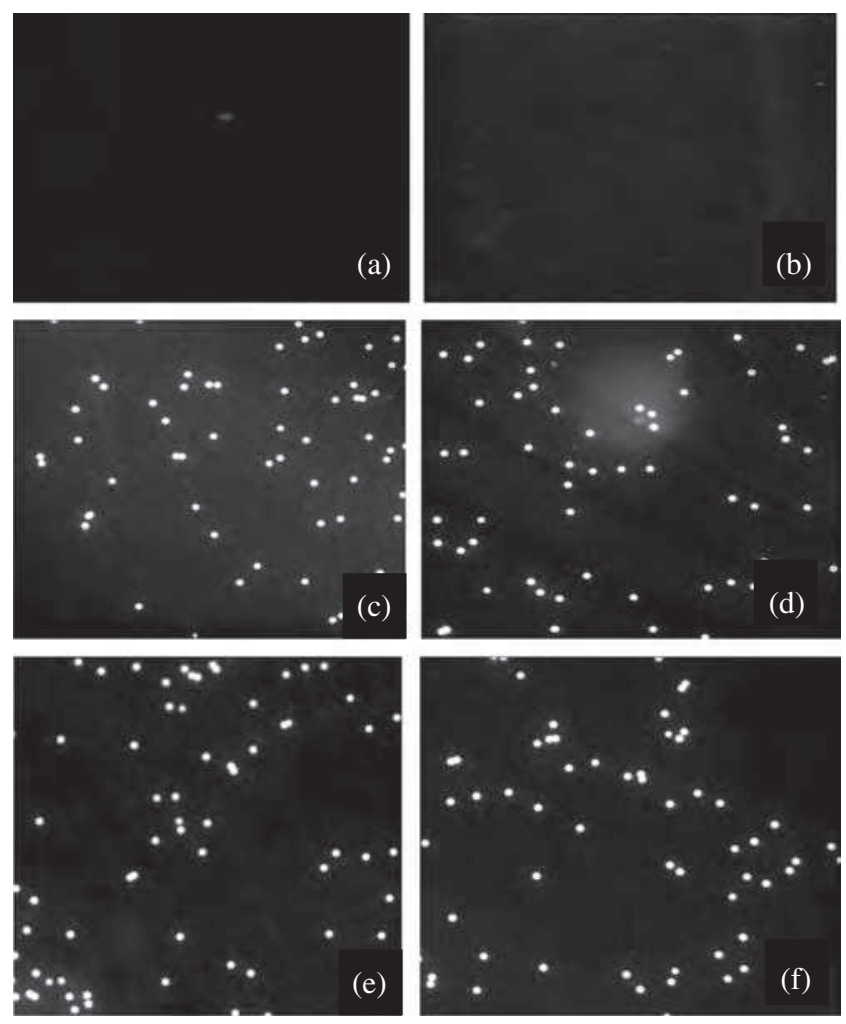

Figure 1. Stability of (poly)bis-SorbPC coated $5 \mu \mathrm{m}$ silica microspheres to extended storage at $4^{\circ} \mathrm{C}$. Fluorescence images were obtained by incubation the microspheres with a fluorogenic dye, FM1-43. (a) uncoated silica microspheres, (b) (poly)bis-SorrbPC coated silica microspheres before incubation with FM1-43, (c) one week storage, (d) two weeks storage, (e) two months storage, and (f) four months storage.

coated with lipids that were not incubated with FM1-43 (figure S1B) exhibit no fluorescence.

Flow cytometry was also used to follow the adsorption of phospholipids onto silica microspheres and to study their stability for extended storage at $4^{\circ} \mathrm{C}$. Figure 2 represents the histograms obtained from flow cytometry for fluorescent phospholipid, (poly)bisSorbPC/Rh-DPPE, bilayers supported on silica microspheres. All (poly)bis-SorbPC/Rh-DPPE coated silica microspheres show an increase in fluorescence indicating deposition of a lipid membrane (figure 2C and D), whereas bare particles and particles coated with non-fluorescent phospholipid, (poly)bis-SorbPC, exhibit no fluorescence (figure $2 \mathrm{~A}$ and $\mathrm{B}$ ). The fluorescence originating from the microspheres that were coated with (poly)bis-SorbPC consisting of Rh-DPPE lipid appeared at the far right in the histogram plot. The fluorescence from uncoated silica microspheres and from those coated with lipids that don't contain the Rh-DPPE lipid came from light scattered by particles into the detector and is shown at the far left of the histogram plot (figure 2A and B). 
Since a flow cytometer only detects fluorescence from the silica microspheres and not from fluorescently labelled vesicles in solution, the flow cytometry data confirms that the lipids are still adsorbed onto the microspheres after extended storage. Moreover, the resulting histogram shows that the population has very narrow fluorescence distribution, suggesting that the silica microspheres were uniformly coated with phospholipids. ${ }^{10}$ There is a very good similarity between the histograms obtained on the $1^{\text {st }}$ day following polymerization (figure $2 \mathrm{C}$ ) and $30^{\text {th }}$ day after being stored at $4^{\circ} \mathrm{C}$ (figure 2D). This similarity indicates that the lipid bilayer coating was not lost with storage. However, one small peak was observed in the fluorescence distribution for particles stored for a month but still no particles with fluorescence less than that of the main peak were observed indicating complete coating of the microspheres with lipids and the stability of the coating.

Furthermore, the stability of the microspheres towards an organic solvent was also studied by fluorescence

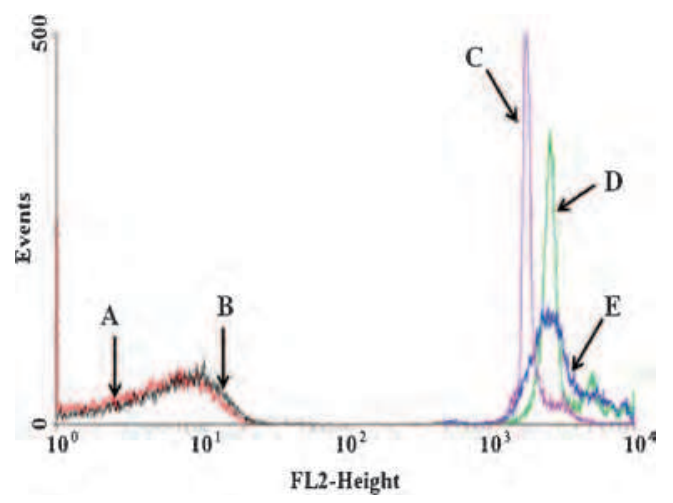

Figure 2. Flow cytometry histograms showing fluorescence distribution of $5 \mu \mathrm{m}$ silica microspheres. (A) uncoated silica microspheres, (B) (poly)bis-SorbPC coated microspheres, (C) (poly)bis-SorbPC/Rh-DPPE coated microspheres after fabrication, (D) (poly)bis-SorbPC/Rh-DPPE coated microspheres stored for one month at $4{ }^{\circ} \mathrm{C}$ and $(\mathrm{E})$ (poly)bis-SorbPC/Rh-DPPE coated microspheres kept dry for 2 weeks. The FL2 stands for fluorescence height recorded in channel 2 of the flow cytometer (arbitrary unit) in log scale. microscopy. Figure S2 in the supplementary information shows the FM1-43 stained lipid coated silica microspheres after washing with a mixture of $\mathrm{ACN}$ and PBS buffer. As can be seen from the figure (right column), unpolymerized phospholipid coated silica microspheres are completely removed from the solid support at $40 \%$ ACN washing The polymerized lipid coated silica particles, however, are found to be stable even to $60 \%$ ACN washing (left column).

3.1b Stability to dry storage: Even though, there are reports on stabilizing supported lipid bilayers to air exposure, there are no reports on the stability of completely dried and rehydrated lipid coated silica particles. Figure 3 shows the FM1-43 stained fluorescence images of phospholipids supported on silica microspheres that have been kept dry for two weeks and rehydrated with buffer. It is important to note that the microspheres were subjected to extensive washing and centrifugation with buffer on the day they are imaged. The lipid coated particles once they are stained with FM1-43 and imaged are discarded to avoid memory effects in subsequent experiments. Aliquots of the coated microspheres that were not treated with FM1-43 were used for future experiments. Though exposure of PLB to drying in air and rehydration of the dried microspheres is expected to cause desorption from the surface, ${ }^{11}$ this was not observed with stabilized (poly)bis-SorbPC coated microspheres. The fluorescence images show that the microspheres are still coated with the PLBs despite dry storage for two weeks indicating the stability of the lipid coating. While not a quantitative measure of the membrane structure, qualitatively the data suggest that the lipids are still deposited onto the silica surface. Though fluorescence microscopy shows no difference between as-prepared and dried phospholipids supported on the microspheres, reorganization of lipids may occur on scales smaller than that can be optically resolved. ${ }^{11}$ However, no fluorescence was observed after four days when unstablized DOPC coated silica

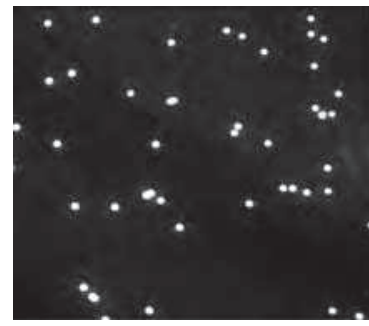

Day 1

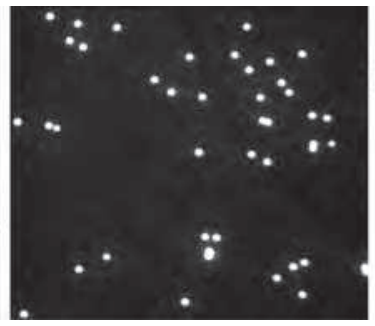

Day 4

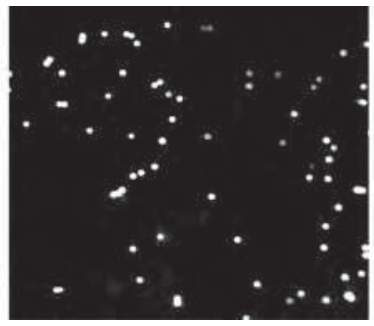

Week 1

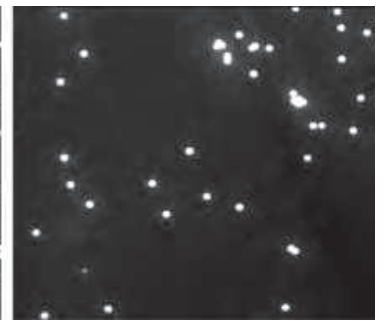

Week 2

Figure 3. Stability of (poly)bis-SorbPC supported on $5 \mu \mathrm{m}$ silica microspheres to dry storage. Fluorescence images were collected after incubation with FM1-43. 
particles were kept dry and subjected to the same rehydration and centrifugation steps (figure 3 and figure S3 in the supplementary information).

There is also a similarity in the fluorescence distribution for microspheres that had been stored for one month at $4{ }^{\circ} \mathrm{C}$ and those stored dry for two weeks (figure 2D and $\mathrm{E}$ ). This similarity in the fluorescence distribution indicates that the coatings are still stable, though they were dried for two weeks. However, the flow cytometry peak is relatively broad for the dried microspheres compared to the ones stored at $4{ }^{\circ} \mathrm{C}$ indicating non-uniform fluorescence intensity in the histogram. This broadening may be the result of formation of an aggregate or restructuring of surface adsorbed phospholipid molecules. However, no microspheres with fluorescence below the main peak was observed suggesting there were no partially or incompletely coated microspheres. The stability of these coatings are in agreement with recent reports by Adem et al. that showed fused silica capillaries coated with (poly)bis-SorbPC are stable for 45 days. ${ }^{12}$

\subsection{Functionalization of phospholipid coated silica}

Deposition of lipid bilayers on silica particles allows the incorporation of biorecognition elements such as biotin, glycolipids and transmembrane protein receptors. Biofunctionalization allows the membrane-coated substrates to be used for detection in a variety of bioassays, including flow cytometry-based assays for cellular receptors.

In this report, silica microspheres were functionalized with DOGS-NTA-Ni ${ }^{2+}$ lipid, to immobilize Histagged proteins. The same approach has been used to functionalize fused silica capillaries in which chemical patterning inside the fused silica was produced using DOGS-NTA-Ni ${ }^{2+}$ lipids to capture 6xHis-EGFP. ${ }^{13}$ Proteins or peptides are routinely engineered with a short sequence of six adjacent histidine residues (6xHis) for immobilization on a solid support. Different surfaces including gold, ${ }^{14,15}$ glass ${ }^{16}$ and polymers ${ }^{17}$ in combination with self-assembled thiol molecules, silanes and surfactants, respectively have been used for immobilization of His-tagged proteins. Silica surfaces modified
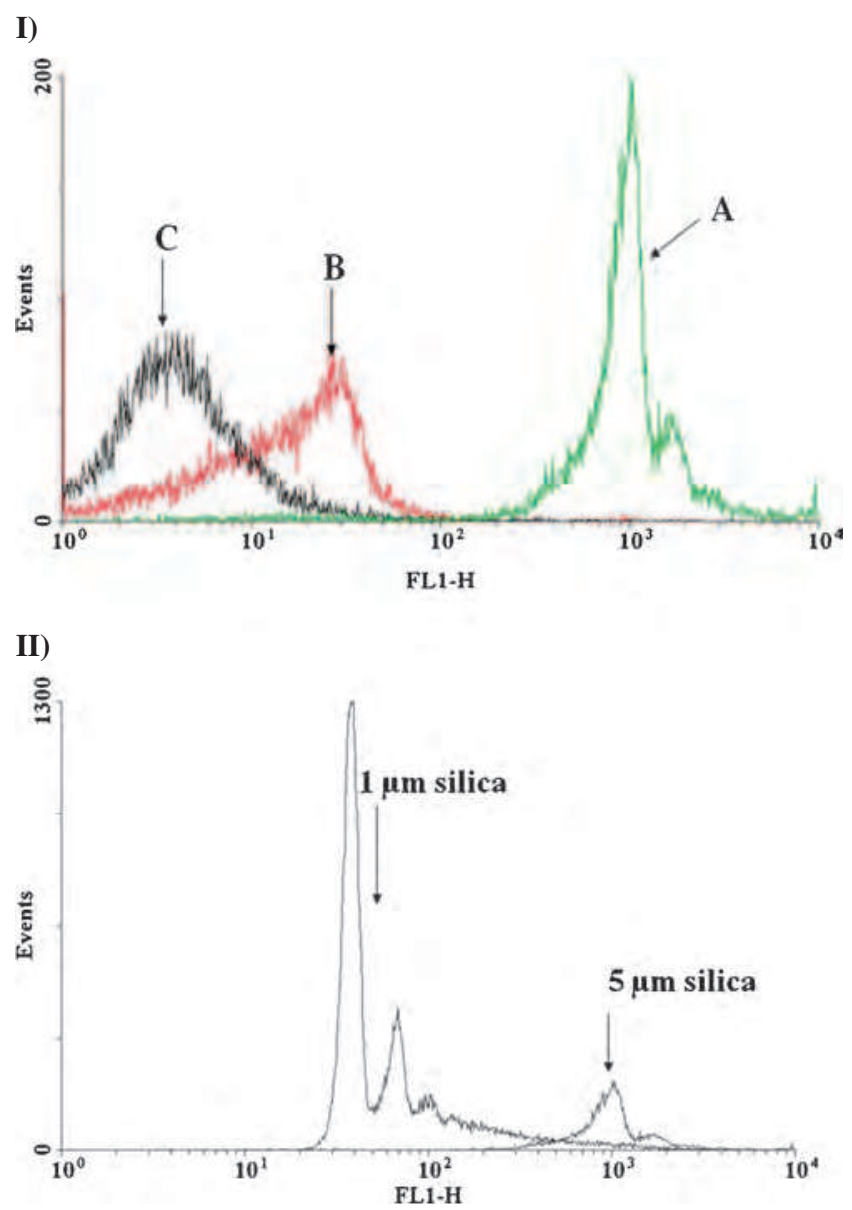

Figure 5. Flow cytometry histograms: (I) obtained from (poly)bis-SorbPC/DOGS-NTA-Ni ${ }^{2+}$ coated $5 \mu \mathrm{m}$ silica microspheres following incubation with 6xHis-EGFP(A), 6xHis-EGFP and washed with $150 \mathrm{mM}$ imidazole (B), and EGFP lacking the His-tag (C); (II) that show the effect of size of the microspheres on the amount of immobilized 6xHisEGFP on DOGS-NTA-Ni ${ }^{2+}$ functionalized microsphere. The FL1-H stands for fluorescence height recorded in channel 1 of the flow cytometer (arbitrary unit) in log scale.
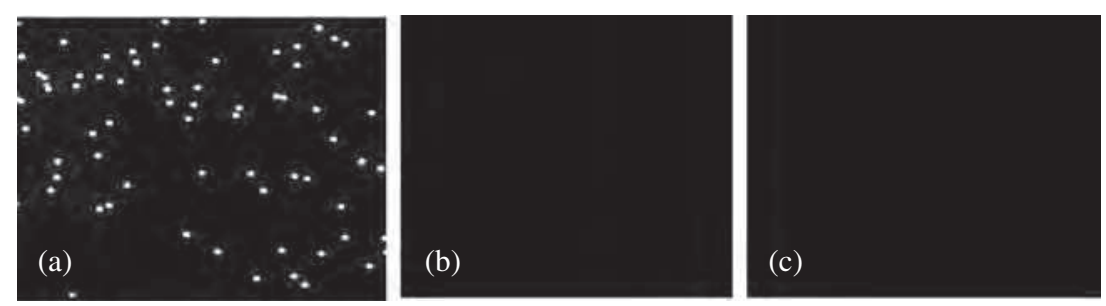

Figure 4. Functionalization of (poly)bis-SorbPC coated $5 \mu \mathrm{m}$ silica microspheres with DOGS-NTA-Ni ${ }^{2+}$. Fluorescence images were collected following incubation with: (a) 6xHis-EGFP, (b) 6xHis-EGFP and washed with $150 \mathrm{mM}$ imidazole and (c) EGFP lacking the His-tag. The corresponding white images are shown in Figure S4 in the supplementary information. 
with polyethylene glycol that was derivatized with $\mathrm{Ni}^{2+} /$ NTA have also been employed for specific adsorption of His-tagged proteins. ${ }^{18}$ However, these techniques are time consuming and laborious as they involve the synthesis of a metal chelator such as NTA or IDA and their attachment to the solid surface. Several techniques such as total internal reflection fluorescence (TIRF), ${ }^{16}$ surface plasmon resonance (SPR), ${ }^{14,15}$ bioluminescence ${ }^{17}$ and atomic force microscopy (AFM $)^{19}$ have been employed to study the binding of His-tagged molecules to a solid surface.

Here, fluorescence microscopy and flow cytometry are employed to follow the functionalization of silica microspheres. Upon incubation with 6xHis-EGFP, bright fluorescent microspheres were observed upon the capture of the protein (figure 4a). When the 6xHis-EGFP incubated functionalized lipid coated silica microspheres were exposed to $150 \mathrm{mM}$ imidazole, the fluorescence intensity of the microspheres decreased to a large extent confirming the reversibility of the interaction (figure 4b). To investigate the specificity of this interaction, EGFP that lacks the 6xHis tag was added to solution of functionalized lipid coated

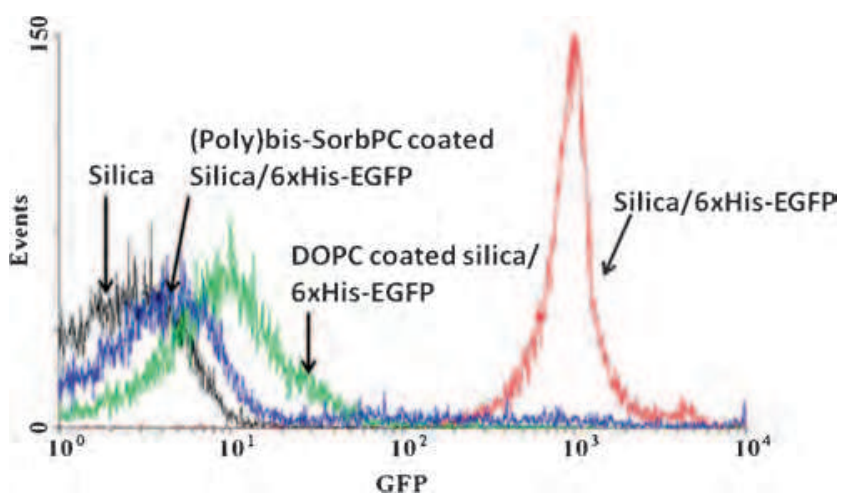

Figure 6. Non-specific protein adsorption: Flow cytometry histograms of 6xHis-EGFP obtained under various conditions. The size of silica microspheres used in this study is $5 \mu \mathrm{m}$. The $\mathrm{x}$-axis represents GFP fluorescence intensity (arbitrary unit) in log scale. microspheres and incubated for $30 \mathrm{~min}$, no fluorescence was observed indicating the absence of interaction with the functionalized silica surface (figure 4c). These observations were further confirmed by flow cytometry data. Figure 5 shows the fluorescence histograms obtained from flow cytometry of silica microspheres coated with bilayers bearing DOGS-NTA-Ni ${ }^{2+}$ that have been incubated with 6xHis-EGFP or EGFP. When silica microspheres functionalized with DOGS-NTA$\mathrm{Ni}^{2+}$ were incubated with $6 x$ His-EGFP, an increase in fluorescence of the microspheres was observed upon capture of the protein (figure 5 IA). The fluorescence intensity is diminished (shifted to the left in the fluorescence histogram) considerably upon washing with imidazole of the microspheres that have been incubated with 6xHis-EGFP (figure 5 IB) and further shifted to the left when the microspheres were incubated with EGFP (figure 5 IC). Furthermore, the amount of immobilized 6xHis-EGFP increases with increasing in the size of the silica microspheres as indicated by the shift to the right in fluorescence distribution for the $5 \mu \mathrm{m}$ silica microspheres relative to the $1 \mu \mathrm{m}$ microspheres (figure $5 \mathrm{II}$ ). This observation is in agreement with previous reports. ${ }^{20,21}$

\subsection{Non-specific protein adsorption}

Improved surface biocompatibility via coating with stabilized polymeric PLB has gained increasing interest since it offers enhanced resistance to non-specific protein adsorption. PC based PLBs have been employed to minimize nonspecific adsorption of proteins and other biological molecules on both planar surfaces and fused silica capillaries. ${ }^{14}$ To evaluate the capability of these (poly)lipid coated silica microspheres to minimize nonspecific protein adsorption, 6xHis-EGFP was incubated with both bare silica and lipid coated silica microspheres. The suspensions were then centrifuged and washed with buffer multiple times to remove excess protein. As shown in figure 6, a high fluorescence was
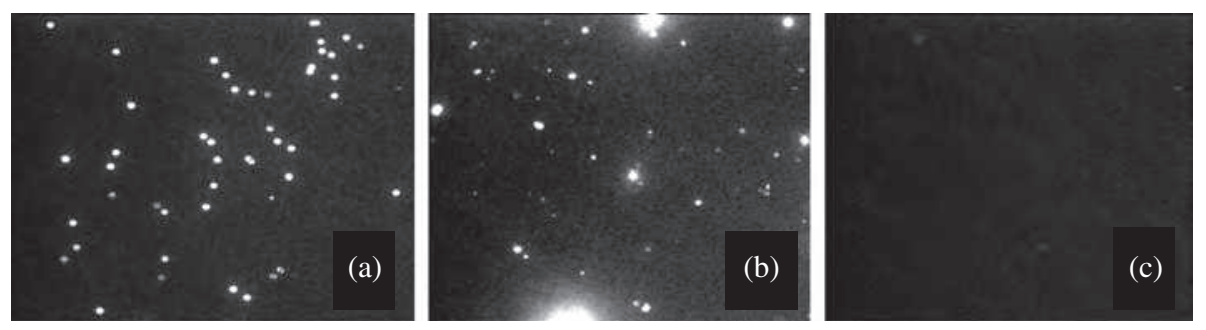

Figure 7. Non-specific protein adsorption: Fluorescence images of 6xHis-EGFP obtained from: (a) bare silica microspheres, (b) DOPC coated silica microspheres and (c) (poly)bis- SorbPC coated silica microspheres. The size of silica particles used for imaging is $5 \mu \mathrm{m}$. The corresponding white images are shown in figure S5 in the supplementary information. 
observed at the right of the histogram for bare particles that have been incubated with the protein, indicating the adsorption of the protein to the silica surface. This fluorescence was decreased and shifted to the left, low fluorescence region, with both DOPC and (poly)bisSorbPC coated silica particles. This observation suggests that in addition to the added benefit of enhanced stability, (poly)bis-SorbPC coating effectively reduces nonspecific adsorption of proteins, though residual nonspecific adsorption is still observed with DOPC coated silica microspheres (figures 6 and 7b). It is likely that some DOPC was removed from the silica upon repeated centrifugations and washings exposing the silica surface to the protein, which may explain the observed fluorescence with DOPC coated particles. As a control, a suspension of bare silica particles not exposed to protein was used and no fluorescence was observed as shown in the histogram. The data from the fluorescence imaging (figure 7c) also supports this observation.

\section{Conclusions}

This report shows the formation of PLBs on silica microspheres using the polymerizable lipid, bis-SorbPC. The resulting polymeric lipid coated silica microspheres were found to be stable to repeated washings and centrifugations, extended storage both in dry (drying/rehydration cycles) and in solution, and to high concentration of organic solvent. Moreover, (poly)lipid coated silica microspheres display a high degree of resistance to non-specific protein adsorption. The ability of the (poly)lipid coatings to be functionalized can be used to modify the charge or binding capabilities of the silica surface without compromising the lipid bilayer structure. Biofunctionalization of the silica microspheres can be used as a model for cellular interfaces and would allow incorporation of membrane proteins. This approach will present an opportunity to develop a novel stationary phase for affinity chromatography to study physiologically and pharmaceutically important small molecules for high-throughput target drug screening.

\section{Supplementary Information}

Fluorescence images showing: the stability of (poly) bis-SorbPC coated silica microspheres to extended storage at $4{ }^{\circ} \mathrm{C}$ and dry storage and acetonitrile washing; functionalization with DOGS-NTA- $\mathrm{Ni}^{2+}$ and non-specific protein adsorption of (poly)bis-SorbPC coated silica microspheres are presented in the supplemental information available at www.ias.ac.in/chemsci

\section{Acknowledgements}

The author would like to thank the Cellular Imaging Facility Core of the University of Arizona for the flow cytometry experiments.

\section{References}

1. Gilbert G E, Drinkwater D, Barter S and Clouse S B 1992 J. Biol. Chem. 26715861

2. Fan L Q, Gioannini T L, Wolinsky T, Hiller J M and Simon E J 1995 J. Neurochem. 652537

3. Devanathan S, Yao Z P, Salamon Z, Kobilka B and Tollin G 2004 Biochemistry 433280

4. Salamon Z, Cowell S, Varga E, Yamamura H I, Hruby V J and Tollin G 2000 Biophys. J. 792463

5. Horie M, Yanagisawa H and Sugawara M 2007 Anal. Biochem. 369192

6. Kloda A, Lua L, Hall R, Adams D J and Martinac B 2007 Proc. Natl. Acad. Sci. U. S. A. 1041540

7. Sukharev S I, Blount P, Martinac B and Kung C 1997 Annu. Rev. Physiology $\mathbf{5 9} 633$

8. Loidl-Stahlhofen A, Kaufmann S, Braunschweig T and Bayerl T M 1996 Nat. Biotechnol. 14999

9. Lamparski H, Liman U, Barry J A, Frankel D A, Ramaswami V, Brown M F and O'Brien D F 1992 Biochemistry 31685

10. Gilbert G E, Drinkwater D, Barter S and Clouse S B 1992 J. Biol. Chem. 26715861

11. Deng Y, Wang Y, Holtz B, Li J, Traaseth N, Veglia G, Stottrup B J, Elde R, Pei D, Guo A and Zhu X Y 2008 J. Am. Chem. Soc. 1306267

12. Adem S M, Mansfield E, Keogh J P, Hall H K and Aspinwall C A 2013 Anal. Chim. Acta 77293

13. Ross E E, Mansfield E, Haung Y and Aspinwall C A 2005 J. Am. Chem. Soc. 12716756

14. Sigal G B, Bamdad C, Barberis A, Strominger J and Whitesides G M 1996 Anal. Chem. 68490

15. Stora T, Dienes Z, Vogel H and Duschl C 2000 Langmuir 165471

16. Schmidt E, Keller T A, Dienes Z and Vogel H 1997 Anal. Chem. 691979

17. Ho C H, Limberis L, Caldwell K D and Stewart R J 1998 Langmuir 143889

18. Kang E, Park J W, McClellan S J, Kim J M, Holland D P, Lee G U, Franses E I, Park K and Thompson D H 2007 J. Am. Chem. Soc. 2316281

19. Raedler U, Mack J, Persika N, Jung G and Tampe R 2000 Biophys. J. 793144

20. Fischer N O, Blanchette C D, Chromy B A, Kuhn E A, Segelke B W, Corzett M, Bench G, Mason P W and Hoeprich P D 2009 Bioconjugate Chem. 20460

21. Lauer S A and Nolan J P 2002 Cytometry 48136 